
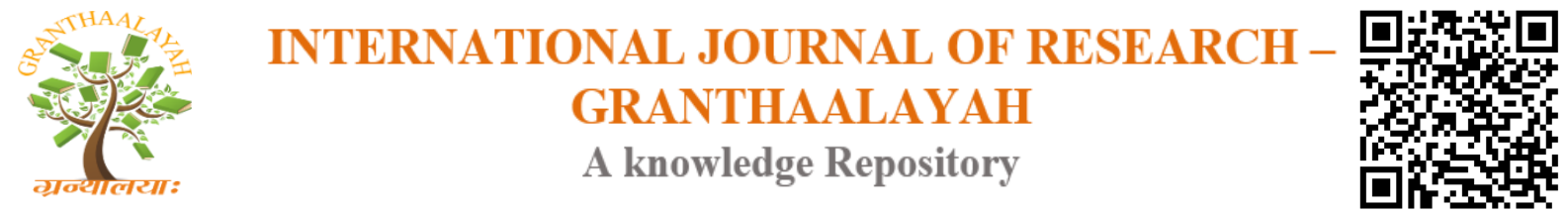

Science

\title{
RAPID LISTERIA MONOCYTOGENES ASSAY BASED ON HELICASE DEPENDENT AMPLIFICATION (HDA) AND NUCLEIC ACID HYBRIDIZATION IN BLUE SILVER NANOPLATES
}

\author{
Kankanit Pisamayarom $^{1,2}$, Piyasak Chaumpluk ${ }^{* 1}$ \\ ${ }^{*}$ Laboratory of Plant Transgenic Technology and Biosensor, Department of Botany, Faculty of \\ Science, Chulalongkorn University, Bangkok 10330, Thailand \\ ${ }^{2}$ Programs in Biotechnology, Faculty of Science, Chulalongkorn University, Bangkok 10330, \\ Thailand
}

\begin{abstract}
Listeria monocytogenes, a foodborne pathogen, is considered as one of the major problems in food safety. With strong safety regulations, a monitoring measure is essential for protecting the health and safety of consumers. Thus, a reliable monitoring method is required. In this study, a rapid assay based on a combination of helicase dependent amplification (HDA) and DNA signal detection via nucleic acid hybridization in blue silver nanoplates (AgNPls) was established. The assay started directly after short term enrichment in terrific broth using cotton ball swapping technique on seafood surface. A HDA amplification of hly gene of L. monocytogenes at $65{ }^{\circ} \mathrm{C}$ allowed DNA signals to be increased, whereas the rendered DNA products were detected via nucleic acid hybridization with an oligonucleotide probe in AgNPls solution. The positive specimens induced blue silver nanoplates' aggregation resulting in pale gray change to colorless, while the negative specimens showed the blue color of non-aggregated nanoplates. The method had a detection limit at 100 copies of L. monocytogenes DNA per $50 \mathrm{~g}$ of sample. This method was rapid, simple, did not require laboratory facilities and was suitable for field food safety monitoring.
\end{abstract}

Keywords: Listeria Monocytogenes; Helicase Dependent Amplification (HDA); Blue Silver Nanoplates (Agnpls); Frozen Seafood.

Cite This Article: Kankanit Pisamayarom, and Piyasak Chaumpluk. (2017). "RAPID LISTERIA MONOCYTOGENES ASSAY BASED ON HELICASE DEPENDENT AMPLIFICATION (HDA) AND NUCLEIC ACID HYBRIDIZATION IN BLUE SILVER NANOPLATES." International Journal of Research - Granthaalayah, 5(10), 322-335. https://doi.org/10.29121/granthaalayah.v5.i10.2017.2308.

\section{Introduction}

Listeria monocytogenes, a gram-positive, causes serious invasive disease or listeriosis in humans and animals. Its ability to survive in a wide range of temperatures and $\mathrm{pH}$ levels, and be able to 
reproduce itself even at low temperatures (Low and Donachie, 1997; Acha and Szyfres, 2003), made it wildly distributed in various environments including soil, silage and in foods. Raw and processed food samples, including dairy products, meat, vegetables and seafood have been reported as common sources of contamination (Gravani, 1999; Nørrung, et al., 1999, Kathariou, 2003). Recently, with a change in our lifestyle, ready-to-eat foods have been reported as a source of listeriosis outbreak (Huss, et al., 2000; de Valk, et al., 2001; Makino, 2005; Tham, et al., 2000).

Infections by ingestion of food contaminated by L. monocytogenes cause severe complications such as non-bloody diarrhea, nausea and vomiting, high fever, and serious septicemia and meningitis (Bortolussi, 2008; Kalorey, 2008). Thus, several regulations were strongly imposed toward strict standards and food safety measures (Jørgensen and Huss, 1998). These include a zero-tolerance in ready-to-eat (RTE) foods for the United Stated and a setting of acceptable levels at $100 \mathrm{CFU} / \mathrm{g}$ for the EU (Jørgensen and Huss, 1998). To implement these regulations at the industry level, self-regulation in risk analysis can be exercised via enforcing a regular screening or monitoring to comply with the Hazard Analysis and Critical Control Point (HACCP). Thus, a rapid reliable assay for detection of this foodborne pathogen is needed.

Until now, a standard culture method using selective agar plates and biochemical and serological identification and modern Polymerase Chain Reaction (PCR) has routinely been used due to its acceptable accuracy; however, implementation of these tests at the on-site level was limited due to them being laborious, complicated, time-consuming and heavily dependent on laboratory facilities (Donelly, 1991) (Furrer, et al., 1991; Bickly, et al., 1996; Bessesen, et al., 1990; Border, et al., 1990; Thomas, et al., 1991).

An ideal assessment method would allow rapid analysis in a single step, enabling the assay to be performed rapidly on site. To achieve this, we have applied a nucleic acid detection combining the DNA amplification and detection steps in sequence. For DNA amplification, Helicase Dependent Amplification (HDA) was selected because it has similar principles to that of PCR (Vincent, et al., 2004), except for the use of thermophillic helicase enzyme to unwind doublestranded DNA instead of heating. It also provided several advantages over PCR such as its rapidity, its equal sensitivity, specificity and its condition involved with the use of isothermal temperature conditions enable it to perform DNA amplification without the need of a thermocycler. These advantages were suitable for the point-of-care application in field. For DNA detection, a colorimetric detection based on metal nanoparticles was employed due to its simplicity and rapidity, providing a result in terms of a direct visual detection without the requirement for any complicated equipment (Giljohann, et al., 2010; Han, et al., 2006).

Previously, unmodified gold nanoparticles had been applied for rapid foodborne pathogen detection, especially for L. monocytogenes and Salmonella enterica (Fu, et al., 2013). However, using gold nanoparticles had its own limitation, especially in terms of their storage stability and the difficult usage due to the requirement in functionalization. These confined their hard-tomanage use to specific conditions, i.e., tests having to be performed rapidly using fresh gold nanoparticles, or the use of nanoparticle functionalization requiring special experiences (Kreibig and Genzel, 1985). These limitations reduce the chance for the method to be applied in real applications. Thus, instead of gold, blue silver nanoplates (AgNPls) with an oligonucleotide 
probe was applied to develop colorimetric detection. The use of blue AgNPls not only solved the stability problem during metal nanoparticle usage but also reduced the cost of the assay and provided clearer colorimetric signals.

In this study, the DNA amplification via helicase dependent amplification (HDA) platform and the DNA hybridization using oligonucleotide in non-functionalized blue silver nanoplates (AgNPls) was applied for Listeria monocytogenes detection in a simple colorimetric assay platform. The use of it for on-site frozen seafood products screening was also demonstrated.

\section{Materials and Methods}

\subsection{Frozen Seafood Specimens and Bacteria Strains}

A total of 30 frozen seafood and ready-to-eat samples were collected from modern type supermarkets and local weekend markets in Bangkok, Thailand, focusing on salmon, tuna, shrimp and their products. All were analyzed after collection in a day. The standard strains of $L$. monocytogenes and non- $L$. monocytogenes were from reliable sources including the Department of Medical Sciences at the Ministry of Public Health, Thailand (DMST), and the Food Research and Testing Laboratory (FRTL) at the Faculty of Science, Chulalongkorn University, Bangkok, Thailand. Detail of strains of pathogenic bacteria including 5 strains of L. monocytogenes and 17 strains of non-L. monocytogenes including L. innocua, L. ivanovii, L. welshimeri of the same genus, Vibrio cholera, V. parahaemolyticus, Salmonella enteritidis, Escherichia coli O157:H7, Enterotoxigenic E. coli (ETEC), Enteropathogenic E. coli (EPEC), Pseudomonas putida, Shigella flexneri, Campylobacter jejuni and Staphylococcus aureus) are described in Table 1 and Table 3.

Table 1: The strains of pathogenic bacteria and sources

\begin{tabular}{|c|c|c|}
\hline No. & Bacteria Strain & Source \\
\hline 1 & Listeria monocytogenes & DMST* 17303 \\
\hline 2 & L. monocytogenes & DMST $^{*} 1327$ \\
\hline 3 & L. monocytogenes & DMST* 20093 \\
\hline 4 & L. monocytogenes & DMST* 23145 \\
\hline 5 & L. monocytogenes & DMST $^{*} 31802$ \\
\hline 6 & L. innocua & DMST $^{*} 9011$ \\
\hline 7 & L. innocua & FRTL** 1265 \\
\hline 8 & L. innocua & FRTL** 1445 \\
\hline 9 & L. innocua & FRTL** 1446 \\
\hline 10 & L. ivanovii & FRTL** 1243 \\
\hline 11 & L. ivanovii & DMST $^{*} 9012$ \\
\hline 12 & L. welshimeri & DMST $^{*} 20559$ \\
\hline 13 & Vibrio cholera & FRTL $* * 1322$ \\
\hline 14 & $V$. parahaemolyticus & FRTL** 0886 \\
\hline 15 & Salmonella enteritidis & DMST $^{*} 15676$ \\
\hline 16 & Escherichia coli $\mathrm{O} 157: \mathrm{H} 7$ & DMST* 12743 \\
\hline 17 & E. coli $(\mathrm{ETEC})$ & DMST* 30543 \\
\hline 18 & E. coli (EPEC) & DMST* 30546 \\
\hline
\end{tabular}


19 Pseudomonas putida

20 Shigella flexneri

21 Campylobacter jejuni

22 Staphylococcus aureus
DMST* 16074

DMST* 4423

DMST* 15190

DMST* 8840

* DMST $=$ Department of Medical Sciences, Ministry of Public Health, Thailand

** FRTL $=$ Food Research and Testing Laboratory, Faculty of Science, Chulalongkorn University, Thailand

\subsection{Genomic DNA Extraction}

For standard samples, DNA extraction was carried out from the overnight bacteria in LB using the Wizard ${ }^{\circledR}$ resin method as described by manufacturer protocol (Promega, USA). For seafood samples, the bacteria were acquired using the cotton ball swab technique from the surface of each sample and enrich-cultured in $2.5 \mathrm{ml}$ Terrific Broth at $37{ }^{\circ} \mathrm{C}$ for $3.5 \mathrm{hrs}$ (Chaumpluk and Chaiprasart, 2013; Sambrook, et al., 1989). The resulting culture was collected in a $1.5 \mathrm{ml}$ microfuge tube, centrifuged at 6,000 rpm for 2 minutes, and the supernatant was discarded. Only $0.25 \mu \mathrm{L}$ of the obtained pellet was used directly for amplification without DNA extraction.

\subsection{Primer Design and Probe}

The hly gene encoding for listeriolysin O, with DNA sequences (GenBank, Accession No. AF253320.1) was employed as reference, and primers were designed using Primer3 (version 0.4.0). In order to confirm their binding specificity, target sequences were aligned using the ClustalW program (Higgins, 1994) and compared against databanks via the Basic Local Alignment Search Tool (BLASTn) (Altschul, et al., 1990). For comparative study between the HDA assay and classical PCR, standard PCR primers as described were used (Furrer, et al., 1991). An oligonucleotide probe specifically targeting the hly gene was also designed for nucleic acid hybridization.

\subsection{Polymerase Chain Reaction (PCR) and Cloning}

Genomic DNA from positive sample was amplified using the PCR technique with specific primers, HDAhlyF and HDAhlyR, and hlyF and hlyR, as described in Table 2 (Furrer, et al., 1991). Obtained products were cloned using TA cloning technique and transformed into E. coli competent cells (SG13009). Plasmid was screened (Sambrook, et al., 1989) and used as positive DNA sample.

\subsection{Isothermal DNA Amplification of $\mathrm{Hly}$ Gene and DNA Signal Detection}

Isothermal DNA amplification based on Helicase dependent amplification (HDA) was employed. Genomic DNA and positive control DNA were amplified using IsoAmp ${ }^{\circledR}$ II Universal tHDA kit (BioHelix, Beverly, MA, USA) with both specific HDA primers via twostep tHDA (thermophilic HDA). In detail, the reaction mixture A was heated for 2 min at $95{ }^{\circ} \mathrm{C}$, and, after adding Mixture B, further incubated at $65^{\circ} \mathrm{C}$ for $40 \mathrm{~min}$. The resulting DNA products were separated on $2.5 \%$ agarose gel and visualized under UV light and ethidium bromide staining. All strains of L. monocytogenes and non-L. monocytogenes DNA were amplified using HDA technique and compared with results from a classical PCR method (Furrer et al., 1991). 
DNA detection was also measured using a colorimetric assay with blue silver nanoplates (AgNPls), 40-120 nm in diameter, synthesized as described (Parnklang, et al., 2013).

The blue AgNPls and the mixing between the oligonucleotide probe and HDA products were performed on the surface of the nanoplates (Chaumpluk, et al., 2013). DNA signals were read after adding salt, which triggered nanoplate aggregation. Briefly, $10 \mathrm{pmol}$ of the oligonucleotide probe were mixed with $1 \mu \mathrm{l}$ of 100x dilution of the HDA products and heated at $80^{\circ} \mathrm{C}$ for 2 min for DNA hybridization. Then $15 \mu \mathrm{l}$ of blue AgNPls were added to the reaction. The color detection of blue AgNPls was observed after adding $1.5 \mu \mathrm{l}$ of $50 \mathrm{mM}$ PBS, pH 7.0 using the naked eye and UV-visible spectroscopy (Ocean Optics ${ }^{\circledR}$ USB2000, USA) at $\lambda=380-800 \mathrm{~nm}$.

\subsection{The Specificity and Sensitivity of HDA}

The specificity of HDA was assayed using genomic DNA of L. monocytogenes and non- $L$. monocytogenes (Table 1) as a template and a specific set of hly primers using the IsoAmp® II kit. The DNA products were then checked by both colorimetric detection and agarose gel electrophoresis. The results were compared in terms of differences of the HDA assay and PCR (Furrer, et al., 1991). The sensitivity of the HDA method was tested using the serial dilution of the plasmid harboring hly gene from 0 to $10^{7}$ copies as described (Kuribara, et al., 2002). The specificity and sensitivity of the method was evaluated (Fawcett, 2006). The specificity was described as the number of true negatives x 100 divided by the sum of the number of false positives and true negatives. The sensitivity was calculated from the number of true positives $\mathrm{x}$ 100 divided by the sum of the number of true positives and false negatives (Yano, et al., 2007).

For the spiking trial, a strain of L. monocytogenes (DMST 17303) was cultured in LB at $37{ }^{\circ} \mathrm{C}$ for 16-18 hrs in a shaking incubator. The serial dilutions of bacteria were determined. Each of the diluted bacteria was inoculated on $50 \mathrm{~g}$ of seafood products and incubated at $37{ }^{\circ} \mathrm{C}$ for 15 min. Then bacteria on sample were obtained by swapping with a sterile cotton ball and enriching for $3.5 \mathrm{hrs}$ at $37^{\circ} \mathrm{C}$. The bacteria culture was harvested as previously described, and measured for the amount of bacteria via plating $50 \mu \mathrm{l}$ of the bacterial serial dilution onto LM media and incubating at $37{ }^{\circ} \mathrm{C}$ for $16-18 \mathrm{hrs}$. The numbers of colonies were calibrated into an original bacteria contamination.

\section{Results and Discussions}

\subsection{DNA Amplification}

Traditional methods for detection for Listeria spp. not only require 5 official days to obtain the result, but rely heavily on laboratory facilities. For this reason, a number of alternative rapid screening tests have been proposed (Välimaaa and ElinaVirtanen, 2015; Liua, et al., 2015; Rodríguez-Lázaro, et al., 2004; Janzten, 2006). The PCR based screening platform and immune chromatographic platform were among the popular models employed recently, but both had drawbacks in terms of being time consuming and depending heavily on laboratory facilities, plus the latter had some problem with test specificity. In this study, the HDA platform was combined with a novel colorimetric DNA hybridization and subsequent nanoparticle aggregation. 
To establish DNA amplification, several candidate genes of L. monocytogenes, including hly, iap, inlA, inlB, prfA and $\operatorname{act} \mathrm{A}$, were investigated. Among them, the $h l y$ gene was selected as the target sequence because it was highly conserved among different strains. The hly gene encodes a hemolysin called listeriolysin O (Cossart and Mengaud, 1989) that is essential for the lysis of the phagosomal membrane during infection. This gene was also the most commonly employed in several molecular analyses (Furrer, et al., 1991; Portnoy, et al., 1988; Churchill, et al., 2006; Jallewar, et al., 2007). To this, a specific set of primers for L. monocytogenes (hly gene) detection was designed (Table 2). The primers were screened based on the BLASTn program on 8 June 2015 (Reference number RGUEX4PY014 and RGUVTT4Y014). The amplification of the hly gene with the primer set using HDA delivered DNA products at 91 nucleotide size. The amplification with HDA provided results (Figure 1A) in agreement with those obtained by classical PCR having 234 nucleotide size (Furrer, et al., 1991).

Table 2: Oligonucleotide sequence for amplification assay of L. monocytogenes

\begin{tabular}{llll}
\hline $\begin{array}{l}\text { Primer } \\
\text { name }\end{array}$ & Primer sequence (5'-3') & $\begin{array}{l}\text { Size } \\
\text { product (bp) }\end{array}$ & $\begin{array}{c}\text { of } \\
\text { Position* }\end{array}$ \\
\hline hlyF $* *$ & 5'-CGG AGG TTC CGC AAA AGA TG-3' & 234 & $1044-1036$ \\
hlyR ** & 5'-CCT CCA GAG TGA TCG ATG TT-3' & & $1258-1277$ \\
HDAhlyF & 5'-TAC CAC GGA GAT GCA GTG AC-3' & 91 & $232-251$ \\
HDAhlyR & 5'-TGG ATT TCT TCT TTT TCT CCA CA-3' & $300-322$ \\
Probe & 5'-CGCCAAGAAAAGGTTACAAAGATGGAAATG-3' & 30 & $260-289$ \\
\hline
\end{tabular}

* With reference to sequence Accession Number AF253320.1 (GenBank)

** Furrer, et al. (1991)

A

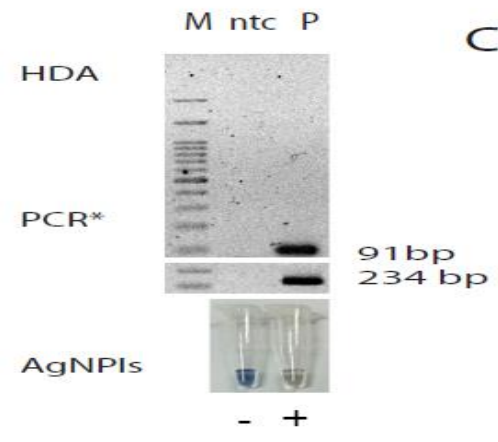

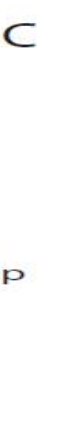

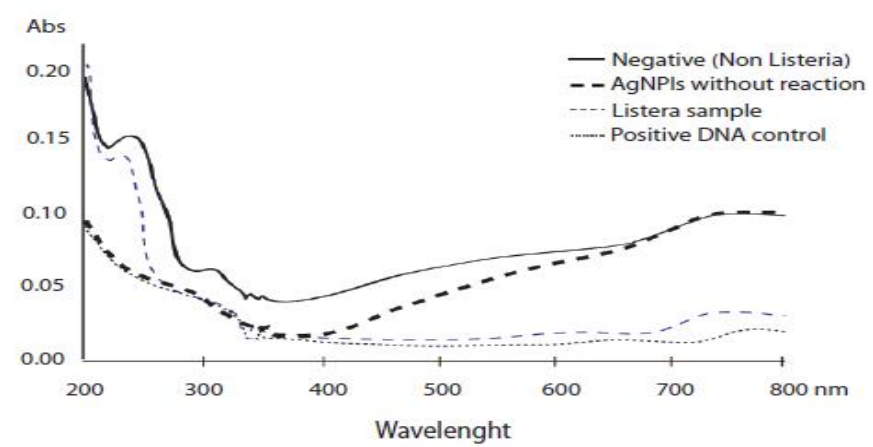

B

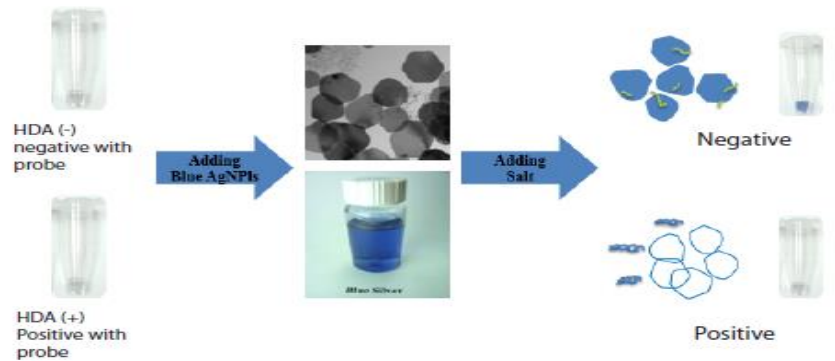

Figure 1: (A) The DNA products of the hly gene using HDA compared with the products from the amplification of the hly gene by PCR (Furrer, et al, 1991) with specific primers and detected based on color changes in the blue AgNPls. Lane M, 100 base pair DNA ladder; Lane ntc, nontemplate control; Lane P, Positive DNA from L. monocytogenes. (B) DNA signal detection based on the control of particle dispersion and aggregation using HDA products and oligonucleotide probe for differentiating between single stranded and double stranded oligonucleotides and (C) 
Absorption spectra of the aggregated blue AgNPls (positive) and dispersed blue AgNPls (negative) by UV-vis spectroscopy at wavelengths from 400-800 $\mathrm{nm}$.

For the primer specificity study, attempts to amplify different DNA templates from 5 strains of L. monocytogenes and 17 strains of non-L. moncytogenes including L. innocua, L. ivanovii, $L$. welshimeri of the same genus, Vibrio cholera, V. parahaemolyticus, Salmonella enteritidis, Escherichia coli O157:H7, Enterotoxigenic E. coli (ETEC), Enteropathogenic E. coli (EPEC), Pseudomonas putida, Shigella flexneri, Campylobacter jejuni and Staphylococcus aureus, using HDA were made. Only the target DNAs from L. monocytogenes were amplified compared with that of PCR. DNA products were amplified using gel electrophoresis and colorimetric detection based on blue silver nanoplates. Only the strains of L. monocytogenes were observed (Figure 2). When non-L. monocytogenes strains were used as template, neither non-specific amplification nor primer dimers were observed. The amplification of the hly gene using specific primer thus occurred only when L. monocytogenes was present. Results also confirmed primer specificity with L. monocytogenes but not L. innocua, L. ivanovi and L. welshimeri, three other species in the same $L$. genus. The results of primer specificity was also in consistent with those studies using the PCR technique (Furrer, et al., 1991), indicating the reliability of primer for Listeria amplification. All these results confirmed the specificity of the HDA primer set for $L$. monocytogenes DNA amplification.

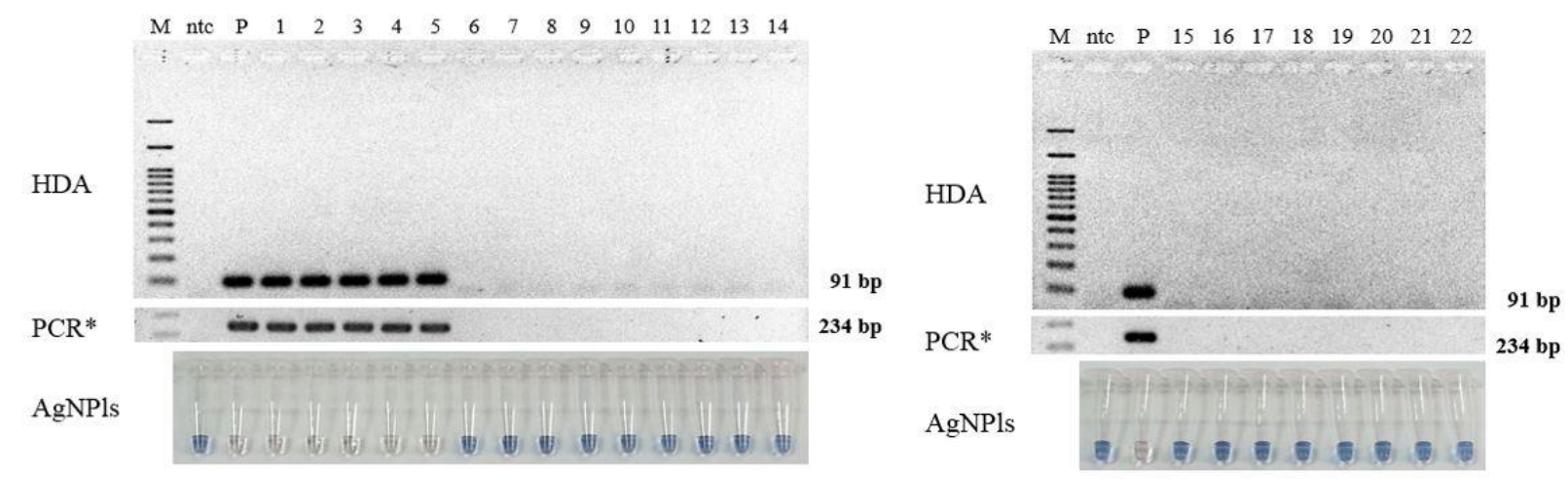

Figure 2: The specificity of the HDA assay for detection of L. monocytogenes compared with PCR and the colorimetric AgNPls. Lane M, 100 base pair DNA ladder; Lane ntc, non-template control; Lane P, positive DNA from L. monocytogenes, Lane 1-5; L. monocytogenes from different source, Lane 6-9, four strains of L. innocua; Lane 10-11, two strains of L. ivanovii; Lane 12-22, L. welshimeri; Vibrio cholera; V. parahaemolyticus; Salmonella enteritidis; Escherichia coli O157:H7; E. coli (ETEC); E. coli (EPEC); Pseudomonas putida; Shigella flexneri; Campylobacter jejuni and Staphylococcus aureus respectively.

The detection limit of HDA primers was also carried out using plasmid harboring the hly gene with ten-fold dilution as described (Kuribara, et al., 2002). The amplification with HDA provided $h l y$ gene products at size of 91 base pairs even when the template was diluted to 100 copies (Figure 3). In our attempt to compare the limit of detection using the PCR technique (Furrer, et al., 1991), it was found that the DNA products size 234 nucleotides were found only when the plasmid templates were diluted to 1000 copies. Since the study was based on purified plasmid template, results then indicated the possibility of better sensitivity for the HDA primer than the PCR. 


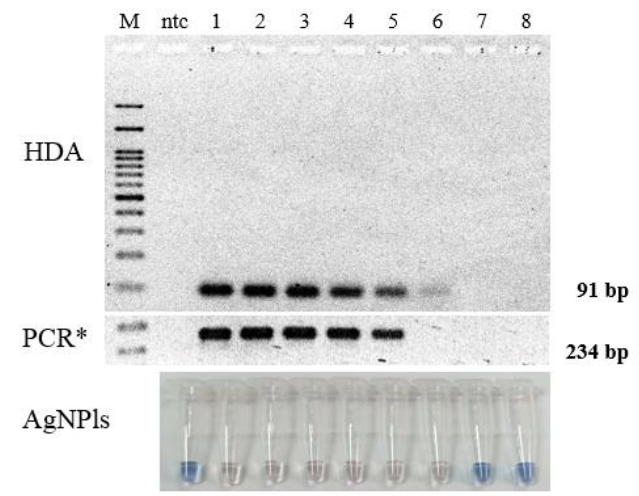

Figure 3: The sensitivity of the HDA assay for detection of L. monocytogenes compared with PCR and the colorimetric AgNPls; Lane M, 100 base pair DNA ladder; Lane ntc, non-template control; Lanes 1-8, ten fold serial dilutions of L. monocytogenes template DNA, ranging from Lane $1,10^{7}$ copies; Lane 2, $10^{6}$ copies; Lane 3, $10^{5}$ copies; Lane 4, $10^{4}$ copies; Lane 5, $10^{3}$ copies; Lane $6,10^{2}$ copies; Lane 7, 10 copies to Lane 8, 0 copies.

Sensitivity of the primer was also investigated based on the spiking of tenfold serially diluted bacterial cultures on $50 \mathrm{~g}$ of frozen seafood together with colony forming plate count evaluation. Results revealed that the limit of detection was approximately $100 \mathrm{CFU}$ in contaminated frozen seafood, which was in agreement with the results obtained from the DNA test (Figure 4). Based on the above, the limit of detection of the primer for Listeria detection was as low as 100 copies of the hly template.

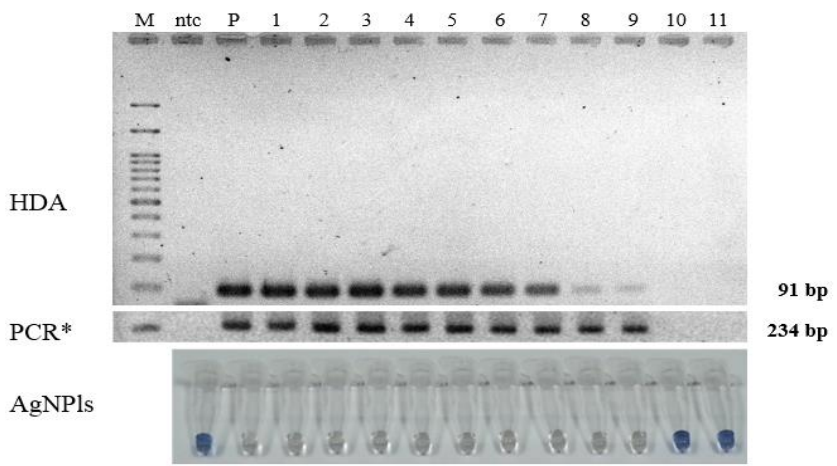

Figure 4: The detection limit by spiking of the seafood sample with L. monocytogenes diluents using an HDA assay compared with PCR and the colorimetric AgNPls; Lane M, 100 base pair DNA ladder; Lane ntc, non-template control; Lane P, L. monocytogenes plasmid, Lane 1-5, from $10^{10}$ to $10^{6} \mathrm{CFU}$, Lane $6,1.02 \times 10^{5} \mathrm{CFU}$, Lane 7, $10250 \mathrm{CFU}$, Lane 8, $1033 \mathrm{CFU}$, Lane 9, 100 CFU, Lane 10, 7 CFU and Lane 11, 0 CFU, respectively.

\subsection{DNA Signal Detection}

The DNA signal detection was based on a colorimetric assay using non functionalized metal nanoparticles due to its advantages in speed and simplicity of the test (Li and Rothberg, 2004). Moreover, the colorimetric assay this way rendered the results observable without instrumentation. The original platform depended on the electrostatic property difference between ssDNA (primers without PCR products) and dsDNA (eg the PCR products). The structure of 
ssDNA allows its configuration to uncoil and expose its bases, whereas that of dsDNA has a stable double-helix geometry, always presenting the negatively charged phosphate backbone (Bloomfield, et al., 2000). The stabilized gold tended to adsorb negative ions whose repulsion prevents the strong van der Waals attraction between the particles from causing them to aggregate (Hunter, 2001). Repulsion between the charged phosphate backbone of dsDNA and the adsorbed citrate ions dominates the electrostatic interaction resulting in unabsorbing on the gold surface. Adsorption of ssDNA stabilizes the gold nanoparticles against salt-induced aggregation and the gold colloid remained ruby red, but this was not the case for dsDNA, where salt-induced aggregation occurred and the gold colloid turned purple.

Recently, $\mathrm{Fu}$ et al. attempted to combine DNA signals detection using a modified gold nanoparticles strategy via a thiolized primer molecule. When the 5, thiol labeling of the oligonucleotide primer was used for hly gene amplification in the PCR platform, the result of amplified DNA products yielded the DNA with thiol groups at 5' terminus. The obtained molecules then attached to the particle via the strong interaction between the thiol groups and the gold, creating thick barriers of a chain of negative charges that protected the gold nanoparticles from salt-induced aggregation.

However, the functionalization of the gold with the thiol group was complicated, laborious and had less storage stability. Moreover, the functionalization efficiency was partially dependent on the reaction conditions, i.e., the $\mathrm{pH}$, electrolyte concentration, heat and magnetic field (Zhou, et al., 2009). These obstacles limited the applicability for on-site testing.

In this study, short oligonucleotide (30 nucleotides) was used as an oligoprobe and performed a colorimetric assay based on non-functionalized, blue-colored silver nanoplates (AgNPls). The use of non-functionalized nanoparticles avoided the problems associated with thiol-group labeling, while blue AgNPls were used instead of gold nanoparticles because they provided longterm stability during a year of storage, unlike the 1-2 month stability of gold nanoparticles. These particles also had a 100 times greater molar extinction coefficient than gold, thus increasing the sensitivity of the assay (Kreibig and Genzel, 1985). The blue AgNPls also provided more clearly visible signals upon aggregation of them, transitioning from blue to colorless, than did the common yellow AgNPls used by Thomson's group, which switched from pale yellow to pale pink (Thompson, et al., 2008). The colorimetric change in the blue AgNPls could be observed by UV-vis spectroscopy at wavelengths from 400-800 nm (Figure 1C). Color changes in the AgNPl solution were observed in $L$. monocytogenes-positive samples but not in samples lacking $L$. monocytogenes.

The effect of signal detection was based on the control of particle dispersion and aggregation using target DNA products and oligonucleotide probes. When the oligonucleotide probes were added at the first step to the diluted HDA products and performed denaturation by heat incubation at $65{ }^{\circ} \mathrm{C}$ for $5 \mathrm{~min}$, the binding of HDA products with probes would result in two different conditions (Figure 1B).

For the positive HDA sample, a large amount of target double stranded DNAs of the hly gene, produced by HDA technique, had opened up a lot of target binding domains which attracted the affinity and consumed a large amount of probes. Shorter oligonucleotide had a tendency to bind 
to target DNA before the longer DNA counterparts. These hybridizations affected the available amount of single strand oligoprobes in the system to less molar level. In general, the single strand oligoprobe tended to bind dispersedly on surface of AgNPls via nitrogenous base/silver van der Waals forces and protected AgNPls from salt induced aggregation ( $\mathrm{Li}$ and Rothberg, 2004). When the probes were totally consumed through the nucleic acid hybridization as that found in the hly gene of the HDA positive case, there were less available probes to protect nanoplates' aggregation in the second step, thus, resulting in the stacking of plates after adding of salt which turned the color of the colloid AgNPls from blue to pale gray to colorless at the end. These color changes could be detected by the naked eye. In the negative HDA sample, there was no DNA amplification and no related products available at all. Then, the adding of the diluted HDA products in the second step did not interfere with the molar amount of single strand oligoprobes in the system, leading to a complete protection of AgNPls from aggregation and inducing no such color change as in scheme 1 . In the test, with $1 \mu 1$ of diluted HDA products, a suitable amount of probes was at 10 pmole, the amount of blue AgNPls (200 ppm) was at $15 \mu 1$ and the amount of salt (100 mM PBS pH 7.0) solutions was $1.5 \mu 1$. These rendered particles aggregation which in turn provided a colorimetric change of AgNPls' colloid from blue to pale gray to colorless in positive, while no such a change was observed in the non-aggregated negative one. Thus, the positive DNA of the hly gene was observed in aggregated (pale gray) of the Blue AgNPls and the negative DNA was still observed in the blue color of the nonaggregation blue AgNPls because the single strand of oligonucleotide probes prevented the blue AgNPls from aggregation in high saline solutions.

The specificity and sensitivity of the assay via colorimetric assay using AgNPls also agreed with those obtained by gel electrophoresis. However, it should be noted that in the case of lower levels of DNA template (diluted DNA template less than 100 copies), the test provided less amplification, which produced insufficient DNA products to induce nanoplate aggregation. The sensitivity test was also carried out using living bacteria as the template. The result on the limits of detection via both nanoplate aggregation and gel electrophoresis was also observed when living bacteria were diluted to $100 \mathrm{CFU}$.

To evaluate the liability of the method, HDA with colorimetric detection was employed for the 100 samples of blind seafood product test. All were directly detected using an HDA assay with specific designed primer and colorimetric detection based on blue AgNPls. Among them, 40 samples were obtained with spiking of known L. monocytogenes concentration and 60 samples were obtained without $L$. monocytogenes contamination. The PCR method was used as standard reference. The spiking concentration of L. monocytogenes was near the limit of detection. The assays results using PCR and HDA with AgNPls were shown in the table 3. The specificity and sensitivity of the detection were calculated as $100 \%$ and $97.5 \%$ respectively, thus this assay is a good candidate for screening and monitoring bacterial contamination in the field.

Table 3: The comparison of PCR with HDA assays using blue silver nanoplates

\begin{tabular}{lll}
\hline Specimens & PCR & HDA with AgNPIs \\
\hline Validation test & & \\
Positive & 40 & 39 \\
Negative & 60 & 61 \\
False positive & - & -
\end{tabular}




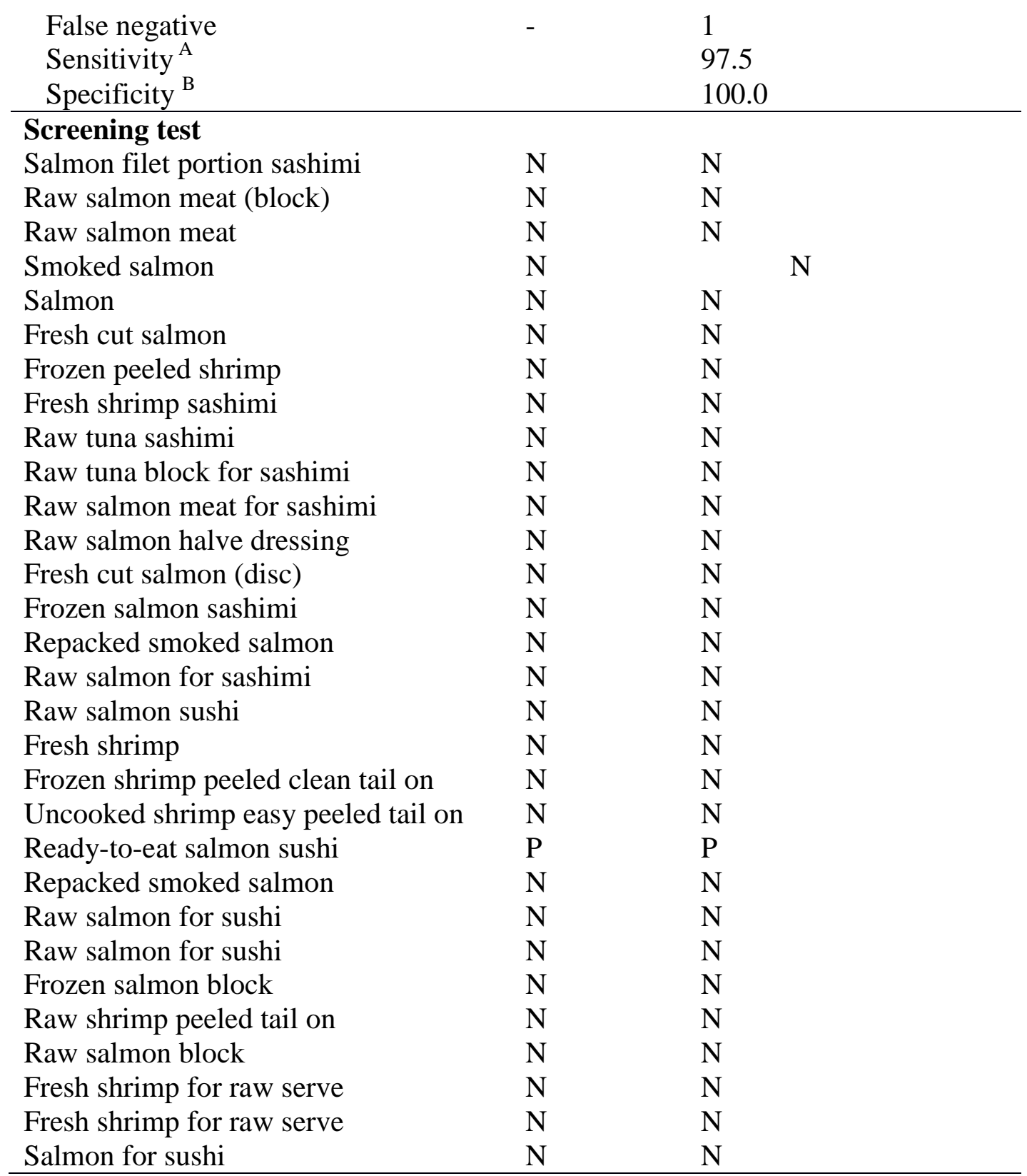

A; Sensitivity $=100 x$ true positive/(true positive + false negative)

$\mathrm{B}$; Specificity $=100 \mathrm{x}$ true negative/(false positive + true negative $)$

$\mathrm{N}$ : negative and P: positive

Finally, rapid screening of frozen seafood in the market (Table 3) was also tested using both PCR and our assay. The results confirmed that a contamination was present in the ready-to-eat salmon sushi from one vendor having bad sanitation (3.33\%). Hence, the standard recommendation was introduced to this vendor. Thus, this test provided a visual readout based on color changes in the silver nanoparticles upon salt-induced aggregation during DNA hybridization. Although this assay is qualitative, the total cost was as low as approximately US\$ 5 per test, and the entire procedure required less than $4.5 \mathrm{hrs}$ to complete $(3.5 \mathrm{hrs}$ for enrichment 
and $\sim 50 \mathrm{~min}$ for the L. monocytogenes assay). This test provides an alternative for monitoring this pathogen in the field, especially for food quality and safety purposes.

\section{Conclusions and Recommendations}

The rapid detection of the hly gene from L. monocytogenes in frozen seafood based on Helicase Dependent Amplification (HDA) and the utilization of blue silver Nanoplates (AgNPls) were developed. The designed primer of the hly gene was specific only for L. monocytogenes and the amplification using an HDA assay has the detection limit of the hly gene at 100 copies of the target gene. This assay provided $100 \%$ specificity and $97.5 \%$ sensitivity. The result could be checked simply from the color change with the naked eye. The total cost was about 5US\$ per test and the process to complete the assay took only $4.5 \mathrm{hrs}$.

\section{Acknowledgements}

The authors would like to thank The Food Research and Testing Laboratory, Faculty of Science, Chulalongkorn University, Bangkok for the support with the types of strains of bacteria. Gratitude is also due to the Faculty of Agriculture Natural Resources and Environment, Naresuan University, Phitsanulok, Thailand, for granting a scholarship to K.P. This work was supported by the Agricultural Research Development Agency (Public Organization). All primers design and methodology/procedures have been submitted to the Patent Registration Office, Ministry of Commerce, and Kingdom of Thailand.

\section{References}

[1] Acha, PN, Szyfres, B. Listeriosis. Zoonoses and Communicable Diseases Common to Man and Animals. (3rded.) Washington D.C.: Pan American Health Organization; 2003, 168-179.

[2] Altschul, SF, Gish, W, Miller, W, Myers, EW, Lipman, DJ. Basic local alignment search tool, Journal of Molecular Biology, 215, 1990, 403-410.

[3] Bessesen, MT, Luo, QA, Rotbart, HA, Blaser, MJ, Ellison, RT. Detection of Listeria monocytogenes by using the polymerase chain reaction, Applied and Environmental Microbiology, 56(9), 1990, 2930-2932.

[4] Bickley, J, Short, JK, McDowell, DG, Parkes, HC. Polymerase chain reaction (PCR) detection of Listeria monocytogenes in diluted milk and reversal of PCR inhibition caused by calcium ions, Letters in Applied Microbiology, 22, 1996, 153.

[5] Bloomfield, VA, Crothers, DM, Tinoco, I, Jr. Nuclei Acids: Structures, Properties, and Functions: Sausalito, CA: University Science Books; 2000.

[6] Border, PM, Howard, JJ, Plastow, GS, Siggens, KW. Detection of Listeria species and Listeria monocytogenes using polymerase chain reaction, Letters in Applied Microbiology, 11, 1990, 158-162.

[7] Bortolussi, R. Listeriosis: a primer, Canadian Medical Association Journal, 179, 2008, 795-797.

[8] Chaumpluk, P, Chaiprasart, P. Fluorescence biosensor based on N-(2-Aminoethyl) glycine peptide nucleic acid for a simple and rapid detection of Escherichia coli in fresh-cut mango, Acta Horticulturae, 992, 2013, 551-560.

[9] Churchill, RLT, Lee, H, Hall, JC. Detection of Listeria monocytogenes and the toxin listeriolysin O in food, Journal of Microbiological Methods, 64, 2006, 141-170.

[10] Cossart, P, Mengaud, J. Listeria monocytogenes: A model system for the molecular study of intracellular parasitism, Molecular Biology \& Medicine, 6, 1989, 463-474. 
[11] de Valk, H, Vaillant, V, Jacquet, C, Rocourt, J, Le Querrec, F, Stainer, F, Quelquejeu, N, Pierre, O, Pierre, V, Desenclos, JC, Goulet, V. Two consecutive nationwide outbreaks of Listeriosis in France, October 1999-February 2000, American Journal of Epidemiology, 154(10), 2001, 944950.

[12] Donelly, CW. In: Ryser ET, Marth EH (Eds.). Listeria, Listeriosis, and Food safety, 2nd ed.; Marcel Dekker Inc., New York, N.Y.; 1991, 225-260.

[13] Fawcett, T. An introduction to ROC analysis. Pattern Recogn Lett. 27, 2006, 861-874.

[14] Fu, Z, Zhou, X, Xing, D. Rapid colorimetric gene-sensing of food pathogenic bacteria using biomodification-free gold nanoparticle, Sensors and Actuators B: Chemical, 182, 2013, 633.

[15] Furrer, B., Candrian, U, Hoefelein, C, Luethy, J. Detection and identification of Listeria monocytogenes in cooked sausage products and in milk by in vitro amplification of haemolysin gene fragments, Journal of Applied Microbiology, 70(5), 1991, 372-379.

[16] Giljohann, DA, Seferos, DS, Daniel, WL, Massich, MD, Patel, PC, Mirkin, CA. Gold nanoparticles for biology and medicine, Angewandte Chemie International Edition in English, 49(19), 2010, 3280-94.

[17] Gravani, R. Listeria in food-processing facilities. In Ryser ET and Marth EH (ed.), Listeria, listeriosis, and food safety; Marcel Dekker, New York, N.Y.; 1999, 657-709.

[18] Han, MS, Lytton-Jean, AK, Oh, BK, Heo, J, Mirkin, CA. Colorimetric screening of DNA binding molecules with gold nanoparticle probes; Angewandte Chemie International Edition in English, 45(11), 2006, 1807-1810.

[19] Hunter, RJ. Foundations of Colloid Science; Oxford University Press, New York; 2001.

[20] Huss, HH, Jorgensen, LV, Vogel, BF. Control options for Listeria monocytogenes in sea foods, International Journal of Food Microbiology, 62(3), 2000, 267-274.

[21] Jallewar, PK, Kalorey, DR, Kurkure, NV, Pandeb, VV, Barbuddhe, SB. Genotypic characterization of Listeria spp. isolated from fresh water fish, International Journal of Food Microbiology, 114, 2007, 120-123.

[22] Jørgensen, LV, Huss, HH. Prevalence and growth of Listeria monocytogenes in naturally contaminated seafood, International Journal of Food Microbiology, 42, 1998, 127.

[23] Kalorey, DK, Warke, SR, Kurkure, NV, Rawool, DB, Barbuddhe, SB. Listeria species in bovine raw milk: A large survey of Central India, Food Control, 19, 2008, 109-112.

[24] Kathariou, S. Foodborn outbreaks of listeriosis and epidemic-associated lineages of Listeria monocytogenes. In ME Torrence and RE Isaacson (ed.), Microbial food Safety in Animal Agriculture: Iowa State University Press, Ames, I; 2003, 243-256.

[25] Kreibig, U, Genzel, L. Optical absorption of small metallic particles, Surface Science, 1985, 156, A330-A331.

[26] Kuribara, H, Shindo, Y, Matsuoka, T, Takubo, K, Futo, S, Aoki, N, Hirao, T, Akiyama, H, Goda, Y, Toyoda, M, Hino, A. Novel reference molecules for quantitation of genetically modified maize and soybean, Journal of AOAC International, 85, 2002, 1077-1089.

[27] Li, H, Rothberg, L. Colorimetric detection of DNA sequences based on electrostatic interactions with unmodified gold nanoparticles, Proceedings of the National Academy of Sciences, 101, 2004, 14036-14039.

[28] Low, JC, Donachie, W. A review of Listeria monocytogenes and listeriosis, Veterinary Journal, 153(1), 1997, 9-29.

[29] Makino, SI, Kawamoto, K, Takeshi, K, Okada, Y, Yamasaki, M, Yamamoto, S, Igimi, S. An outbreak of food-borne listeriosis due to cheese in Japan, during 2001, International Journal of Food Microbiology, 104, 2005, 189-196.

[30] Nørrung, B, Andersen, JK, Schlundt, J. Incidence and control of Listeria monocytogenes in foods in Denmark, International Journal of Food Microbiology, 53(2-3), 1999, 195-203.

[31] Parnklang, T, Lertvachirapaiboon, C, Pienpinijtham, P, Wongravee, K, Thamma-charoen, C, Ekgasit, S. H2O2-triggered shape transformation of silver nanospheres to nanoprisms with 
controllable longitudinal LSPR wavelengths, Royal Society of Chemistry Advances, 3, 2013, 12886-12894.

[32] Portnoy, DA, Jacks, PS, Hinrichs, DJ. Role of hemolysin for the intracellular growth of Listeria monocytogenes, Journal of Experimental Medicine, 67, 1988, 1459-1471.

[33] Sambrook, J, Fritsch, EF, Maniatis, T. Molecular cloning: a laboratory manual: Cold Spring Harbor Laboratory Press; 1989.

[34] Tham, W, Ericsson, H, Loncarevic, S, Unnerstad, H, Danielsson-Tham, ML. Lessons from an outbreak of listeriosis related to vacuum-packed gravad and cold-smoked fish, International Journal of Food Microbiology, 62, 2000, 173-175.

[35] Thomas, EJG, King, RK, Burchak, J, Gannon, VPJ. Sensitive and specific detection of Listeria monocytogenes in milk and ground beef with the polymerase chain reaction, Applied and Environmental Microbiology, 57, 1991, 2576-2580.

[36] Thompson, DG, Enright, A, Faulds, K, Smith, WE, Graham, D. Ultrasensitive DNA detection using oligonucleotide-silver nanoparticle conjugates, Analytical Chemistry, 80, 2008, 2805-2810.

[37] Vincent, $\mathrm{M}, \mathrm{Xu}, \mathrm{Y}$, Kong, H. Helicase-dependent isothermal DNA amplification, EMBO Reports, 5(8), 2004, 759-800.

[38] Yano, A, Ishimaru, R, Hujikata, R. Rapid and sensitive detection of heat-labile I and heat-stable I enterotoxin genes of enterotoxigenic Escherichia coli by Loop-Mediated Isothermal Amplification, Journal of Microbiological Methods, 68(22), 2007, 414-420.

[39] Zhou, J, Ralston, J, Sedev, R, Beattie, DA. Functionalized gold nanoparticles: synthesis, structure and colloid stability, Journal of Colloid and Interface Science, 331, 2009, 251-262.

*Corresponding author.

E-mail address: piyasakcha@yahoo.com/toomkankanit@ gmail.com 